\title{
Association Between Sarcopenia and Tooth Loss
}

\author{
Chang Hoon $\mathrm{Han}^{1}$, Jae Ho Chung ${ }^{2}$ \\ ${ }^{1}$ Department of Internal Medicine, National Health Insurance Service Ilsan Hospital, Goyang, Korea \\ ${ }^{2}$ Department of Internal Medicine, International St. Mary's Hospital, Catholic Kwandong University College of Medicine, Incheon, Korea
}

Corresponding Author:

Jae Ho Chung, MD

https://orcid.org/0000-0001-7590-2277

Department of Internal Medicine, International St. Mary's Hospital, Catholic Kwandong University College of Medicine, 25 Simgok-ro 100beongil, Seo-gu, Incheon 22711, Korea

Tel: +82-32-900-2813

Fax: +82-32-900-3879

E-mail: klaatu70@gmail.com

Received: June 19, 2018

Revised: July 20, 2018

Accepted: July 25, 2018
Background: The aim of this study was to determine whether the prevalence and extent of periodontal disease and tooth loss are increased in participants with sarcopenia. Methods: This cross-sectional study included 8,053 males (sarcopenia, 2,772; nonsarcopenia, 5,281) and 10,729 females (sarcopenia, 3,085; nonsarcopenia, 7,644) $\geq 18$ years of age who participated in the Korean National Health and Nutrition Examination Survey (KNHANES) from 2008 to 2011 and underwent assessments of oral health and appendicular skeletal muscle mass. Muscle mass index was calculated as appendicular muscle mass divided by height squared. The cutoff values for sarcopenia were $7.0 \mathrm{~kg} /$ $\mathrm{m}^{2}$ for males and $5.4 \mathrm{~kg} / \mathrm{m}^{2}$ for females. Results: The group with sarcopenia brushed their teeth less frequently. The presence of periodontitis was significantly higher in participants with sarcopenia (males, 30.3\%; females, $45.9 \%$ ) than in participants without sarcopenia (males, 18.3\%; females, 17.4\%) ( $p<0.001)$. The number of natural teeth was significantly lower in participants with sarcopenia. The adjusted odds ratio for sarcopenia in participants with <20 natural teeth compared to those with full dentition was 1.96 (95\% confidence interval [Cl], 1.58-2.48) in males and 2.86 (95\% Cl, 2.31-3.56) in females. Subgroup analysis of the adjusted odds ratio for sarcopenia in older ( $\geq 65$ years) individuals with <20 natural teeth was $1.92(95 \% \mathrm{Cl}, 1.49-2.66)$ in males and $2.63(95 \% \mathrm{Cl}$, 2.25-3.64) in females. Conclusion: Loss of the natural teeth wass significantly associated with sarcopenia. (Ann Geriatr Med Res 2018;22:145-150)

Key Words: Oral health, Sarcopenia, Tooth

\section{INTRODUCTION}

Tooth loss is caused by complex interactions among several risk factors. ${ }^{1)}$ Clinical dental factors such as dental caries and periodontal status are linked directly, ${ }^{2)}$ and other factors, such as health behaviors and general health problems, are indirectly linked to tooth loss. ${ }^{3)}$ Tooth loss leads to a poor chewing ability, ${ }^{4,5)}$ which can alter dietary habits and the oral-health-related quality of life. ${ }^{6)}$ Demographic factors, such as age, sex, household income, educational status, smoking, alcohol drinking, and frequency of tooth brushing ${ }^{7)}$ have also been associated with tooth loss. ${ }^{8)}$ Sarcopenia, defined as a decreased muscle mass or function, is associated with the aging process, ${ }^{9)}$ as is tooth loss in many older individuals.

Many reports have demonstrated the associations between oral health status and systemic health. Tooth loss is an objective indicator of oral health. Chronic diseases such as coronary heart disease ${ }^{10)}$ and diabetes mellitus (DM) ${ }^{11)}$ are well-known risk factors for tooth loss. Sarcopenia and tooth loss, which are common features in elderly individuals, might be accompanied by other risk factors such as
DM. Although an accurate assessment of muscle mass requires expensive equipment and may therefore not be feasible in community settings, from a public health standpoint, it is important to perform careful assessment of the possible relationship between tooth loss and sarcopenia if either is to be managed appropriately. Therefore, the aim of this study was to investigate whether sarcopenia is associated with tooth loss.

\section{MATERIALS AND METHODS}

\section{Study Participants}

This study acquired data from the Korean National Health and Nutrition Examination Surveys (KNHANES) from 2008 to 2011. KNHANES is a nationwide investigation used to periodically assess the health and nutritional status of noninstitutionalized people in South Korea. From this population, this study included 8,053 males and 10,729 females $\geq 18$ years of age in whom muscle mass and oral health were assessed. Patients who did not undergo any one of these assessments were excluded. 


\section{Baseline Health and Socioeconomic Status}

Household income and educational levels were categorized, as indicators of socioeconomic status. Since muscle mass can be affected by nutritional status, we used data of 24-hour recall of food intake, of which previously shown as a reliable and valid way in assessing nutritional status. ${ }^{12)}$ Smoking, alcohol drinking, and exercise status were assessed. A smoker was defined as someone who had smoked >100 cigarettes in his/her lifetime. Risky drinking was defined as the consumption of $>5$ alcohol beverages at one occasion, and $>12$ alcohol drinks during the last year. Regular exercise was defined according to the American College of Sports Medicine Guidelines. ${ }^{13)}$ DM was determined based on the response to a self-administered questionnaire using the yes-or-no question, "Have you ever been diagnosed with DM by your physician?”

\section{Measurements of Muscle Mass}

Dual energy X-ray absorptiometry (Discovery-W, Hologic Inc.; Waltham, MA, USA) was used to measure muscle mass. Appendicular skeletal muscle mass (ASM) was calculated as the sum of arm and leg muscle mass. Muscle mass index was defined as the ASM divided by height squared (ASM $[\mathrm{kg}] /$ height $[\mathrm{m}]^{2}$ ) below the sex-specific mean of the young reference group (18-40 years of age). ${ }^{14)}$ In our study, a muscle index of $\leq 7.0 \mathrm{~kg} / \mathrm{m}^{2}$ for males and $\leq 5.4 \mathrm{~kg} / \mathrm{m}^{2}$ for females was the cutoff level used to define sarcopenia according to the European consensus on the definition and diagnosis of sarcopenia. ${ }^{15)}$

Table 1. Clinical characteristics of study participants

\begin{tabular}{|c|c|c|c|c|c|c|}
\hline \multirow[b]{2}{*}{ Characteristic } & \multicolumn{2}{|c|}{ Male } & \multirow[b]{2}{*}{ p-value } & \multicolumn{2}{|c|}{ Female } & \multirow[b]{2}{*}{ p-value } \\
\hline & $\begin{array}{c}\text { Sarcopenia } \\
(\mathrm{n}=2,772)\end{array}$ & $\begin{array}{l}\text { Nonsarcopenia } \\
\quad(n=5,281)\end{array}$ & & $\begin{array}{c}\text { Sarcopenia } \\
(n=3,085)\end{array}$ & $\begin{array}{l}\text { Nonsarcopenia } \\
\quad(n=7,644)\end{array}$ & \\
\hline Age (yr) & $55.1 \pm 0.3$ & $46.5 \pm 0.2$ & $<0.001$ & $50.9 \pm 0.2$ & $45.8 \pm 0.3$ & $<0.001$ \\
\hline \multicolumn{7}{|l|}{ Lifestyle factors } \\
\hline Smoking (current smoker) & $596(21.5)$ & $1,201(22.7)$ & 0.354 & $356(11.5)$ & $684(9.0)$ & $<0.001$ \\
\hline Regular exercise & $1,457(52.6)$ & $3,083(58.4)$ & $<0.001$ & $1,432(46.4)$ & $4,037(52.8)$ & $<0.001$ \\
\hline Risky drinking & $1,056(38.1)$ & $1,709(32.4)$ & $<0.001$ & $250(8.1)$ & $563(7.4)$ & 0.109 \\
\hline Family income & & & $<0.001$ & & & $<0.001$ \\
\hline Low & $760(27.4)$ & $739(13.9)$ & & $781(25.4)$ & $1,367(17.9)$ & \\
\hline Moderate-low & $703(25.4)$ & $1,344(25.6)$ & & $619(20.0)$ & $2,047(26.8)$ & \\
\hline Moderate-high & $686(24.7)$ & $1,645(31.1)$ & & $829(26.9)$ & $2,016(26.4)$ & \\
\hline High & $623(22.5)$ & $1,559(29.5)$ & & $856(27.7)$ & $2,214(28.9)$ & \\
\hline Education & & & $<0.001$ & & & $<0.001$ \\
\hline$\leq$ Elementary & 770 (27.9) & $773(14.6)$ & & 909 (29.5) & $1,596(20.9)$ & \\
\hline Middle school & $384(13.8)$ & $608(11.5)$ & & $240(7.8)$ & $880(11.5)$ & \\
\hline High school & 839 (30.9) & $2,105(39.9)$ & & 954 (30.9) & $3,150(41.2)$ & \\
\hline$\geq$ College & $779(27.4)$ & $1,795(34.0)$ & & $982(31.8)$ & $2,018(26.4)$ & \\
\hline \multicolumn{7}{|l|}{ Anthropometric indices } \\
\hline Body mass index $\left(\mathrm{kg} / \mathrm{m}^{2}\right)$ & $21.3 \pm 0.1$ & $25.1 \pm 0.1$ & $<0.001$ & $20.6 \pm 0.1$ & $24.4 \pm 0.1$ & $<0.001$ \\
\hline Waist circumference $(\mathrm{cm})$ & $79.4 \pm 0.2$ & $86.8 \pm 0.1$ & $<0.001$ & $71.9 \pm 0.1$ & $81.3 \pm 0.1$ & $<0.001$ \\
\hline ASM $(\mathrm{kg})$ & $18.9 \pm 0.4$ & $23,7 \pm 0.4$ & $<0.001$ & $12.6 \pm 0.1$ & $15.1 \pm 0.1$ & $<0.001$ \\
\hline $\mathrm{ASM} / \mathrm{ht}^{2}\left(\mathrm{~kg} / \mathrm{m}^{2}\right)$ & $6.98 \pm 0.08$ & $8.24 \pm 0.09$ & $<0.001$ & $5.1 \pm 0.1$ & $6.2 \pm 0.1$ & $<0.001$ \\
\hline Total body fat mass $(\mathrm{kg})$ & $13.5 \pm 0.9$ & $16.4 \pm 0.7$ & $<0.001$ & $16.7 \pm 0.1$ & $19.8 \pm 0.1$ & $<0.001$ \\
\hline Total body fat percentage (\%) & $21.7 \pm 0.1$ & $22.1 \pm 0.1$ & $<0.001$ & $32.5 \pm 0.1$ & $33.1 \pm 0.1$ & $<0.001$ \\
\hline \multicolumn{7}{|l|}{ Nutritional status } \\
\hline Energy intake (kcal/day) & $2073 \pm 17$ & $2414 \pm 15$ & $<0.001$ & $1632 \pm 12.3$ & $1641 \pm 7.6$ & 0.494 \\
\hline Protein (g) & $72.8 \pm 0.8$ & $87.1 \pm 0.7$ & $<0.001$ & $58.1 \pm 0.6$ & $57.3 \pm 0.3$ & 0.098 \\
\hline Calcium (g) & $323.6 \pm 6.7$ & $374.2 \pm 5.7$ & $<0.001$ & $431.5 \pm 5.4$ & $446.9 \pm 4.1$ & 0.037 \\
\hline Carbohydrate (g) & $333.8 \pm 2.3$ & $367.3 \pm 1.9$ & $<0.001$ & $274.7 \pm 1.9$ & $288.2 \pm 1.4$ & $<0.001$ \\
\hline Fat (g) & $37.1 \pm 0.7$ & $49.2 \pm 0.6$ & $<0.001$ & $33.1 \pm 0.5$ & $29.2 \pm 0.3$ & $<0.001$ \\
\hline Diabetes mellitus & $312(11.3)$ & $396(7.5)$ & $<0.001$ & $163(5.3)$ & $624(8.2)$ & $<0.001$ \\
\hline
\end{tabular}

Values are presented as mean \pm standard error or number (\%).

Risky drinking was defined as drinking more than 5 alcoholic beverages at one occasion, and drinking more than 12 drinks at one occasion during the previous year.

Regular exercise was defined as moderate or strenuous exercise performed on a regular basis ( $>30$ minutes at a time 5 times per week of moderate exercise or $>20$ minutes at a time 5 times per week of strenuous exercise, respectively) or walking $>30$ minutes at a time more than 5 times per week.

Daily energy and nutrient intake was assessed using the 24-hour recall method that was used in the nutrition survey.

ASM, appendicular skeletal muscle, sum of both arm and leg. 


\section{Number of Natural Teeth}

The number of natural teeth was investigated, based on the normal complement of 32 teeth and excluding third molars. According to this information, participants were classified into three groups: full dentition (28 teeth), 20-27 teeth, and $<20$ teeth.

\section{Other Oral Health Assessment}

A dentist conducted oral health checkups as part of the survey. Periodontitis was defined using community periodontal index (CPI: CPI 0, healthy teeth; CPI 1, gingival bleeding; CPI 2, calculus; CPI 3, shallow periodontal pocket; and CPI 4, deep periodontal pocket). ${ }^{16)}$ Periodontitis was defined as a CPI of 3 or CPI 4. The mean kappa score for interexaminer reliability was 0.89 (0.55-1.00). The regular use of secondary oral health products such as mouthwash, an interdental brush, dental floss, or an electric toothbrush may help to maintain oral health by reducing the risk of infection. Therefore, this study also investigated the use of these secondary oral health products.

\section{Ethics}

The Korea Centers for Disease Control and Prevention approved the study protocol (approval numbers: 2008-04EXP-01-C, 2009-01CON-03-2C, 2010-02CON21-C, 2011-02CON-06-C).

\section{Data Analysis}

All variables investigated in the present study were stratified using IBM SPSS Statistics ver. 20.0 (IBM Co., Armonk, NY, USA). All clinical characteristics were compared among participants using the chi-square test or Fisher exact test for categorical variables. A multiple logistic regression analysis was also performed, adjusting for age, regular exercise, family income, education, alcohol consumption, smoking, total energy intake, total body fat mass, calcium intake, presence of DM, and presence of periodontitis. Adjustment for socio-economic factors including age, household income, education, behavioral factors including smoking, risky drinking, exercise, total energy intake, total body fat mass, calcium intake and presence of DM, periodontitis, number of tooth brush-

Table 2. Relationship between oral health behaviors and sarcopenia

\begin{tabular}{|c|c|c|c|c|c|c|}
\hline \multirow[b]{2}{*}{ Variable } & \multicolumn{2}{|c|}{ Male } & \multirow[b]{2}{*}{ p-value } & \multicolumn{2}{|c|}{ Female } & \multirow[b]{2}{*}{ p-value } \\
\hline & $\begin{array}{c}\text { Sarcopenia } \\
(\mathrm{n}=2,772)\end{array}$ & $\begin{array}{c}\text { Nonsarcopenia } \\
(\mathrm{n}=5,281)\end{array}$ & & $\begin{array}{c}\text { Sarcopenia } \\
(\mathrm{n}=3,085)\end{array}$ & $\begin{array}{c}\text { Nonsarcopenia } \\
(n=7,644)\end{array}$ & \\
\hline Dental visit in the last year & $628(22.7)$ & $1,350(25.6)$ & 0.013 & $645(21.0)$ & $1,656(21.7)$ & 0.346 \\
\hline Perceived oral health status & & & $<0.001$ & & & $<0.001$ \\
\hline Very good & $29(1.0)$ & $77(1.5)$ & & $216(7.0)$ & $896(11.7)$ & \\
\hline Good & $321(11.6)$ & $690(13.1)$ & & $712(23.1)$ & $1,875(24.5)$ & \\
\hline Moderate & $980(35.3)$ & $2,030(38.4)$ & & $1,051(34.1)$ & $1,991(26.1)$ & \\
\hline Bad & $1,123(40.5)$ & $2,036(38.5)$ & & $750(24.3)$ & $1,898(24.8)$ & \\
\hline Very bad & $319(11.5)$ & $448(8.5)$ & & $356(11.5)$ & 984 (12.9) & \\
\hline Frequency of tooth brushing & & & $<0.001$ & & & $<0.001$ \\
\hline$\leq 1$ time per day & $976(35.2)$ & $829(15.7)$ & & $851(27.6)$ & $924(12.1)$ & \\
\hline 2 times per day & $1,574(56.8)$ & $3,340(63.2)$ & & $1,574(51.0)$ & $4,913(64.3)$ & \\
\hline$\geq 3$ times per day & $222(8.0)$ & $1112(21.1)$ & & $660(21.4)$ & $1,807(23.6)$ & \\
\hline \multicolumn{7}{|l|}{ Use of other oral products } \\
\hline Dental floss & $171(6.2)$ & $491(9.3)$ & 0.001 & $420(13.6)$ & $1,037(13.6)$ & 0.922 \\
\hline Mouthwash & $124(4.5)$ & $393(7.4)$ & 0.030 & $226(7.3)$ & $564(7.4)$ & 0.913 \\
\hline Interdental brush & $229(8.3)$ & 509 (9.6) & 0.102 & $348(11.3)$ & $918(12.0)$ & 0.232 \\
\hline Electric toothbrush & $86(3.1)$ & 384 (7.3) & $<0.001$ & $165(5.4)$ & $346(4.5)$ & 0.069 \\
\hline Total number of teeth & $24.1 \pm 0.1$ & $26.3 \pm 0.1$ & $<0.001$ & $23.3 \pm 0.1$ & $26.4 \pm 0.1$ & $<0.001$ \\
\hline Tooth loss & & & $<0.001$ & & & $<0.001$ \\
\hline No (28 teeth) & $1,173(42.3)$ & $3,240(61.4)$ & & $1,215(39.4)$ & $4,830(63.2)$ & \\
\hline Mild (20-27 teeth) & $1,053(38.0)$ & $1,756(33.3)$ & & $1,268(41.1)$ & $2,352(33.1)$ & \\
\hline Moderate (<20 teeth) & $546(19.7)$ & $285(5.3)$ & & $602(19.5)$ & $282(3.7)$ & \\
\hline DMF (decayed+missing+filled) teeth & $7.0 \pm 0.1$ & $5.7 \pm 0.1$ & $<0.001$ & $8.1 \pm 0.1$ & $5.9 \pm 0.1$ & 0.030 \\
\hline Periodontitis & $839(30.3)$ & $964(18.3)$ & $<0.001$ & $1416(45.9)$ & $1323(17.3)$ & 0.012 \\
\hline Periodontal severity & & & $<0.001$ & & & $<0.001$ \\
\hline Normal & $1,267(45.7)$ & $3,318(62.8)$ & & $1,206(39.1)$ & $4,858(63.6)$ & \\
\hline Gingival bleeding & $476(17.2)$ & $498(9.4)$ & & $310(10.0)$ & $873(11.4)$ & \\
\hline Calculus & $220(7.9)$ & $501(9.5)$ & & $153(5.0)$ & $590(7.7)$ & \\
\hline Shallow periodontal pocket & $505(18.2)$ & $809(15.3)$ & & $839(27.2)$ & $999(13.0)$ & \\
\hline Deep periodontal pocket & $304(11.0)$ & $155(3.0)$ & & $577(18.7)$ & $324(4.3)$ & \\
\hline
\end{tabular}

Values are presented as number (\%) or mean \pm standard deviation. 
ings per day, and use of secondary oral products were performed for determining the odds ratio of tooth loss in sarcopenia.

\section{RESULTS}

The study population comprised 8,053 males and 10,729 females $\geq 18$ years of age in whom muscle mass and oral health were assessed. Individuals who did not undergo either one of these assessments were excluded $(n=7,549)$.

Table 1 lists the clinical characteristics of the study population. Among males, participants in the sarcopenia group were, on average, older than those in the non-low muscle mass group $(55.1 \pm 0.3$ vs. $46.5 \pm 0.2, p<0.001)$. They were also less educated [less than elementary school: 770 $(27.4 \%)$ vs. 739 (13.9\%); $p<0.001$ ], less likely to exercise regularly $(1,457[52.6 \%]$ vs. $3,083[58.4 \%], p<0.001)$, and more likely to have a lower income (760 [27.4\%] vs. 739 [13.9\%], $\mathrm{p}<0.001)$ and a smaller total energy intake. Female participants in the sarcopenia group were, on average, older than those in the nonsarcopenia group $(50.9 \pm 0.2$ years vs. $45.8 \pm 0.3$ years, $\mathrm{p}<0.001$ ) as well as less more educated (less than elementary school: 909 [29.5\%] vs. 1,596 [20.9\%], $\mathrm{p}<0.001)$, less likely to exercise regularly $(1,432$ [46.4\%] vs. 4,037 [52.8\%], $\mathrm{p}<0.001)$, and more likely to have lower income (low income: 781 [25.4\%] vs. 1,367 [17.9\%], $\mathrm{p}<0.001)$. Table 2 shows the relationships among sarcopenia status and oral hygiene and self-care. Periodontitis (defined as a $\mathrm{CPI} \geq 3$ ) was more prevalent in the sarcopenia group (males, 30.3\%; females, 45.9\%) than in the non-sarcopenia group (males, 18.3\%; females, 17.3\%). Additionally, only $22.7 \%$ of male participants in the sarcopenia group had seen a dentist in the last year. Despite these facts, $12.6 \%$ (good, 11.6\%; very good, 1.0\%) of male participants in the sarcopenia group stated that their oral health status was better than good. Male participants with sarcopenia were significantly less likely than those without sarcopenia to brush their teeth three or more times per day $(8.0 \%$ vs. $21.1 \%, p<0.001)$. Male participants with sarcopenia were also significantly less likely than those without sarcopenia to use secondary oral health products. Among both male and female participants, the number of natural teeth was significantly lower in the sarcopenia group than in the nonsarcopenia group. The DMF (decayed + miss- ing + filled teeth) index was also significantly higher in the sarcopenia group for both sexes (males: $7.0 \pm 0.1$ vs. $5.7 \pm 0.1, \mathrm{p}<0.001$; females: $8.1 \pm 0.1$ vs. $5.9 \pm 0.1, \mathrm{p}<0.001$ ). The adjusted odds ratio for sarcopenia in participants with $<20$ natural teeth was 1.96 (95\% confidence interval [CI], 1.58-2.48) for males and 2.86 (95\% CI, 2.31-3.56) for females compared to male and female participants with full dentition (Table 3).

Table 4 shows the results of a subgroup analysis of the adjusted odds ratios of sarcopenia vs. remaining teeth according to age group. The adjusted odds ratio for sarcopenia in participants with $<20$ natural teeth was 1.14 (95\% CI, 1.10-1.97) in young and middle-aged ( $<65$ years of age) male participants and 2.31 (95\% CI, 2.07-3.26) in young and middle-aged ( $<65$ years of age) female participants. The adjusted odds ratio for sarcopenia in participants with <20 natural teeth was 1.92 (95\% CI, 1.49-2.66) in older ( $\geq 65$ years of age) males and 2.63 (95\% CI, 2.263.64 ) in older ( $\geq 65$ years of age) females compared to older male and female participants with full dentition.

\section{DISCUSSION}

This study showed that sarcopenia was associated with tooth loss. Sarcopenia and tooth loss are common problems in the geriatric population. Because tooth loss often leads to decreased oral function such as compromised eating and chewing ability, ${ }^{4,5}$ it eventually reduces muscle mass and thus the quality of life. ${ }^{6)}$ Thus, identifying the risk factors for tooth loss is important to establish a strat-

Table 3. Multivariate adjusted logistic regression analysis between the remaining teeth and sarcopenia

\begin{tabular}{lcc}
\hline Teeth & Male & Female \\
\hline$<20$ & $1.96(1.58-2.48)$ & $2.86(2.31-3.56)$ \\
$20-27$ & $1.20(1.08-1.41)$ & $2.19(1.91-2.51)$ \\
28 & Reference & Reference \\
\hline
\end{tabular}

Values are presented as odds ratio (95\% confidence interval). Adjusted for socio-economic factors including age, household income, education, behavioral factors including smoking, risky drinking, exercise, total energy intake, total body fat mass, calcium intake and presence of diabetes mellitus, periodontitis, number of tooth brushings per day, and use of secondary oral products.

Table 4. Subgroup analysis of adjusted odds ratios of sarcopenia for remaining teeth according to age groups

\begin{tabular}{lccccc}
\hline \multirow{2}{*}{ Teeth } & \multicolumn{2}{c}{ Young and middle age $(<65$ years of age $)$} & & \multicolumn{2}{c}{ Older people $(\geq 65$ years of age) } \\
\cline { 2 - 5 } \cline { 5 - 6 } & Male & Female & Male & Female \\
\hline 20 & $1.14(1.10-1.97)$ & $2.31(2.07-3.26)$ & & $1.92(1.49-2.66)$ & $2.63(2.26-3.64)$ \\
$20-27$ & $1.66(1.50-2.20)$ & $2.17(2.08-2.68)$ & & $1.88(1.62-2.39)$ & $2.47(2.24-2.97)$ \\
28 & Reference & Reference & & Reference & Reference \\
\hline
\end{tabular}

Values are presented as odds ratio (95\% confidence interval).

Adjusted for socio-economic factors including age, household income, education, behavioral factors including smoking, risky drinking, exercise, total energy intake, total body fat mass, calcium intake and presence of diabetes mellitus, periodontitis, number of tooth brushings per day, and use of secondary oral products. 
egy for maintaining oral health.

Studies of the association between weight and tooth loss have reported inconsistent results. Some found an association between being underweight and poor oral health. ${ }^{17)}$ However, Elwood and Bates ${ }^{18)}$ found no differences in tooth loss by body weight, whereas in the series of Halling et al., ${ }^{19)}$ individuals with 1-15 teeth tended to weigh more than those with $>15$ teeth. Some studies have investigated weight change and tooth loss; however, little research is available regarding sarcopenia and tooth loss. ${ }^{20)}$ Low muscle mass may negatively affect both chewing capacity and oral muscle function. A Japanese study found that occlusal power was associated with handgrip strength, walking speed, and body muscle volume. ${ }^{21)}$ A recent longitudinal study ${ }^{22)}$ reported that the loss of oral occlusion power was associated with a decline in leg muscle power. These results support our finding that low muscle mass is related to tooth loss. In a recent study, ${ }^{23)}$ poor oral health, including tooth loss, was shown to be a predictor of future sarcopenia among community-dwelling older adults. Our study is cross-sectional; therefore, future studies should investigate whether sarcopenia affects tooth loss. Although the mechanism remains unclear, associations between tooth loss and low muscle mass may occur via inflammatory ${ }^{24)}$ and nutritional pathways. ${ }^{25)}$ Inflammatory cytokines activate many of the molecular pathways involved in skeletal muscle wasting, leading to an imbalance between protein synthesis and catabolism; this inflammation is a common feature of periodontitis. Tooth loss affects dietary quality and nutrient intake in a manner that may increase the risk for sarcopenia.

Most oral health studies have used periodontal indexes, such as dental probing, to assess dental status. However, the diagnosis of periodontal disease is subjective because of poor interexaminer reliability; thus, it is unclear whether periodontal indexes can objectively assess oral health status for research purposes. Oral health studies differ in their definitions of periodontitis, as well as in their methods for determining periodontal status and cutoffs for periodontal diseases. ${ }^{26)}$ In our study, the kappa value for inter-examiner reliability was 0.89 ; however, in other studies it was generally between 0.7 and 0.8. Different definitions of dental status and different methods for determining periodontal status used in other studies limit comparisons of their results. ${ }^{27,28)}$ In contrast, the measurement of "number of teeth lost" is a reliable indicator of dental health status for both general health clinicians and patients. Therefore, we focused on the associations between low muscle mass and tooth loss instead of the periodontal index.

This study had several limitations. First, it was a crosssectional study and longitudinal relationships between the identified factors could not be determined. Hence, we were unable to assess definitively the cause-and-effect relationships between low muscle mass and tooth loss. Second, our study participants were relatively healthy because in- dividuals admitted to hospitals or nursing homes were not included; thus, low muscle mass may have been underestimated. Third, our study did not take into account dental implants, prostheses, and dentures; therefore, additional evidence on the relationship between tooth loss and low muscle mass is needed.

Despite these limitations, this study benefited from a large sample size and extensive data on potential confounders for sarcopenia and tooth loss, which allowed multiple statistical analyses. These showed that patients with sarcopenia are at increased risk for tooth loss, even in old age.

In conclusion, this cross-sectional study identified an association between tooth loss and sarcopenia.

\section{CONFLICTS OF INTEREST DISCLOSURES}

The researchers claim no conflicts of interest.

\section{ACKNOWLEDGMENTS}

This work was supported by research funding from Catholic Kwandong University International St. Mary's Hospital (CKURF-201601570001).

\section{REFERENCES}

1. Hugo FN, Hilgert JB, de Sousa Mda L, da Silva DD, Pucca GA Jr. Correlates of partial tooth loss and edentulism in the Brazilian elderly. Community Dent Oral Epidemiol 2007;35:224-32.

2. Bahrami G, Vaeth M, Kirkevang LL, Wenzel A, Isidor F. Risk factors for tooth loss in an adult population: a radiographic study. J Clin Periodontol 2008;35:1059-65.

3. Burt BA, Ismail AI, Morrison EC, Beltran ED. Risk factors for tooth loss over a 28-year period. J Dent Res 1990;69:1126-30.

4. Endrikat J, Shapiro H, Lukkari-Lax E, Kunz M, Schmidt W, Fortier M. A Canadian, multicentre study comparing the efficacy of a levonorgestrel-releasing intrauterine system to an oral contraceptive in women with idiopathic menorrhagia. J Obstet Gynaecol Can 2009;31:340-7.

5. Fontijn-Tekamp FA, Slagter AP, Van Der Bilt A, Van 'T Hof MA, Witter DJ, Kalk W, et al. Biting and chewing in overdentures, full dentures, and natural dentitions. J Dent Res 2000;79:1519-24.

6. Stenman U, Ahlqwist M, Björkelund C, Hakeberg M. Oral healthrelated quality of life--associations with oral health and conditions in Swedish 70-year-old individuals. Gerodontology 2012;29:e4406.

7. Hanioka T, Ojima M, Tanaka K, Aoyama H. Association of total tooth loss with smoking, drinking alcohol and nutrition in elderly Japanese: analysis of national database. Gerodontology 2007;24:87-92.

8. Gilbert GH, Duncan RP, Shelton BJ. Social determinants of tooth loss. Health Serv Res 2003;38(6 Pt 2):1843-62.

9. Cruz-Jentoft AJ, Baeyens JP, Bauer JM, Boirie Y, Cederholm T, Landi F, et al. Sarcopenia: European consensus on definition and diagnosis: Report of the European Working Group on Sarcopenia 
in Older People. Age Ageing 2010;39:412-23.

10. Elter JR, Champagne CM, Offenbacher S, Beck JD. Relationship of periodontal disease and tooth loss to prevalence of coronary heart disease. J Periodontol 2004;75:782-90.

11. Kaur G, Holtfreter B, Rathmann W, Schwahn C, Wallaschofski H, Schipf S, et al. Association between type 1 and type 2 diabetes with periodontal disease and tooth loss. J Clin Periodontol 2009; 36:765-74

12. Sun Y, Roth DL, Ritchie CS, Burgio KL, Locher JL. Reliability and predictive validity of energy intake measures from the 24hour dietary recalls of homebound older adults. J Am Diet Assoc 2010;110:773-8.

13. Haskell WL, Lee IM, Pate RR, Powell KE, Blair SN, Franklin BA, et al. Physical activity and public health: updated recommendation for adults from the American College of Sports Medicine and the American Heart Association. Circulation 2007;116:1081-93.

14. Baumgartner RN, Koehler KM, Gallagher D, Romero L, Heymsfield SB, Ross RR, et al. Epidemiology of sarcopenia among the elderly in New Mexico. Am J Epidemiol 1998;147:755-63.

15. Chen LK, Liu LK, Woo J, Assantachai P, Auyeung TW, Bahyah KS, et al. Sarcopenia in Asia: consensus report of the Asian Working Group for Sarcopenia. J Am Med Dir Assoc 2014;15:95-101.

16. World Health Organization. Oral health surveys: basic methods. 5th ed. Geneva (Switzerland): World Health Organization; 2013.

17. Sheiham A, Steele JG, Marcenes W, Finch S, Walls AW. The relationship between oral health status and Body Mass Index among older people: a national survey of older people in Great Britain. $\mathrm{Br}$ Dent J 2002;192:703-6.

18. Elwood PC, Bates JF. Dentition and nutrition. Dent Pract Dent Rec 1972;22:427-9.

19. Halling A, Bengtsson C, Lenner RA. Diet in relation to number of remaining teeth in a population of middle-aged women in Gothenburg, Sweden. Swed Dent J 1988;12:39-45.

20. Murakami M, Hirano H, Watanabe Y, Sakai K, Kim H, Katakura A. Relationship between chewing ability and sarcopenia in Japanese community-dwelling older adults. Geriatr Gerontol Int 2015;15:1007-12.

21. Kono R. Relationship between occlusal force and preventive factors for disability among community-dwelling elderly persons. Nihon Ronen Igakkai Zasshi 2009;46:55-62.

22. Okuyama N, Yamaga T, Yoshihara A, Nohno K, Yoshitake Y, Kimura Y, et al. Influence of dental occlusion on physical fitness decline in a healthy Japanese elderly population. Arch Gerontol Geriatr 2011;52:172-6.

23. Tanaka T, Takahashi K, Hirano H, Kikutani T, Watanabe Y, Ohara Y, et al. Oral frailty as a risk factor for physical frailty and mortality in community-dwelling elderly. J Gerontol A Biol Sci Med Sci 2017 Nov 17 [Epub]. https://doi.org/10.1093/gerona/glx225.

24. El-Shinnawi U, Soory M. Associations between periodontitis and systemic inflammatory diseases: response to treatment. Recent Pat Endocr Metab Immune Drug Discov 2013;7:169-88.

25. Ritchie CS, Joshipura K, Hung HC, Douglass CW. Nutrition as a mediator in the relation between oral and systemic disease: associations between specific measures of adult oral health and nutrition outcomes. Crit Rev Oral Biol Med 2002;13:291-300.

26. Hujoel PP, Drangsholt M, Spiekerman C, DeRouen TA. Periodontal disease and coronary heart disease risk. JAMA 2000;284:1406-10.

27. Machtei EE, Christersson LA, Grossi SG, Dunford R, Zambon JJ, Genco RJ. Clinical criteria for the definition of "established periodontitis". J Periodontol 1992;63:206-14.

28. Beck JD, Löe H. Epidemiological principles in studying periodontal diseases. Periodontol 2000 1993;2:34-45. 